\title{
Comparison of The Variability of Small Extracellular Vesicles Derived From Human Liver Cancer Tissues And Cultured From The Tissue Explants Based On A Simple Enrichment Method: Yield, Purity And Molecular Markers
}

Jie Chen ( $\square$ gnyxycj@163.com )

Gannan Medical College: Gannan Medical University https://orcid.org/0000-0002-1104-8910

\section{Zhigang Jiao}

First Affiliated Hospital Of Gannan Medical College

Jianwen Mo

First Affiliated Hospital Of Gannan Medical College

Defa Huang

Gannan Medical College: Gannan Medical University

Zhengzhe Li

Gannan Medical College: Gannan Medical University

Wenjuan Zhang

First Affiliated Hospital Of Gannan Medical College

Tong Yang

Gannan Medical College: Gannan Medical University

Minghong Zhao

Gannan Medical College: Gannan Medical University

Fangfang Xie

Gannan Medical College: Gannan Medical University

Die Hu

Gannan Medical College: Gannan Medical University

Xiaoxing Wang

Gannan Medical College: Gannan Medical University

Xiaomei Yi

Gannan Medical College: Gannan Medical University

Yu Jiang

University of Pittsburgh School of Medicine

Tianyu Zhong

First Affiliated Hospital Of Gannan Medical College 


\section{Research Article}

Keywords: small extracellular vesicles, exosomes, isolation, purification, liver cancer tissue

Posted Date: July 13th, 2021

DOl: https://doi.org/10.21203/rs.3.rs-680243/v1

License: (c) (i) This work is licensed under a Creative Commons Attribution 4.0 International License. Read Full License 


\section{Abstract}

A potential of using small extracellular vesicle (sEV) for diagnostic and therapeutic purposes has attracted great interest. Some sEVs, directly derived from various tissues, can reflect the physiological and pathological state of the organism. Currently, there are two sources for obtaining tissue-derived sEV: the interstitial space of tissues and cultivation of tissue explants. The former was obtained from tissues that were digested with enzymes and the latter was released by tissue explants. In the present study, we established a new method for the isolation and purification of $\mathrm{SEV}$ s from the interstitial space of human liver cancer tissue. Tissues-derived SEVs (TdsEVs) isolated by this method and cultured explants-derived sEVs (cedsEVs) were both characterized by nano-flow cytometry (NanoFCM) for concentration, size distribution and purity. These vesicles were identified and compared by transmission electron microscopy and western blot. TdsEVs have a larger particle size, higher particle concentration and purity than cedsEVs. This study establishes a simple sEV isolation and purification protocol and provides a basis for selection and reference for researches of tdsEVs and cedsEVs.

\section{Introduction}

sEVs, a kind of bilayer phospholipid membrane vesicles (30-200 $\mathrm{nm}$ in diameter), are secreted by various types of cells and released to extracellular space. The vesicles play a role in communication between donor and recipient cells (1). sEVs exist in a variety of body fluids, such as blood (2), urine (3), amniotic fluid (4) and so on. Their protein and RNA contents can, to certain extent, reflect the physiological and pathological state of the human body, and may serve as disease biomarkers (5).

Liver cancer is the third leading cause of cancer-related deaths worldwide, and most patients are diagnosed at an advanced stage with poor prognosis (6). Recently, an increasing body of studies has focused on analyzing the protein contents from tdsEVs and cedsEVs for diagnosis of tumors. TdsEVs and cedsEVs have the characteristics of complex microenvironment that more closely related to human body and richer sources of information than cell lines, as well as purer exclusiveness than circulating $s E V s$ in body fluids. Therefore, these vesicles are of great research value.

We first identified the types of digestive enzymes in liver cancer tissues. Collagenase $D$ and DNase I have been used to extract sEVs from human metastatic melanoma tissues $(7 ; 8)$, colon cancer tissues and colonic mucosa tissues (9). Collagenase $D$ digests the intercellular matrix and degrades natural collagen with little damage to cells, while DNase I is able to reduce the viscosity of the tissue suspension, hence facilitating isolation of cells (9). Combination of the enzymes results in releasing more tdsEVs. In addition, we determined the optimal conditions for the enzymatic digestion, and compared three different isolation methods for applicability. Our study establishes a simple new method for sEVs preparations. Using this newly developed method, we isolated and analyzed tdsEVs and cedsEVs in terms of particle size distribution, particle concentration, purity and protein markers.

\section{Materials And Methods}




\section{Tissue collection and preparation}

Human liver cancer tissues were collected from patients diagnosed with liver malignancy at the First Affiliated Hospital of Gannan Medical University. Informed written consents were obtained from the patients and the collection of human tissue samples was approved by the Ethics Committee of the First Affiliated Hospital of Gannan Medical University. All samples were transported in pre-cooled PBS. The samples were divided into three parts, which were used for the preparation of fresh tdsEVs (tissue samples collected within $2 \mathrm{~h}$ ), frozen tdsEVs (tissue samples stored at $-80^{\circ} \mathrm{C}$ for at least $24 \mathrm{~h}$ ) and cedsEVs (tissue samples collected within $2 \mathrm{~h}$ ).

\section{Separation and purification of tdsEVs and cedsEVs}

To obtain tdsEVs with high purity, three different methods were used (Fig. 1a). Specifically, the tissues were cut into small pieces $(2 \times 2 \times 2 \mathrm{~mm})$ on ice and weighed, then placed in RPMI- 1640 medium containing collagenase $D(1 \mathrm{mg} / \mathrm{mL}, 2 \mathrm{mg} / \mathrm{mL}$ and $4 \mathrm{mg} / \mathrm{mL})$ and DNase I $(20 \mathrm{U} / \mathrm{mL}, 40 \mathrm{U} / \mathrm{mL}$ and 80 $\mathrm{U} / \mathrm{mL}$ ) and incubated at $37^{\circ} \mathrm{C}$ for $20 \mathrm{~min}$ or (and) $30 \mathrm{~min}$ (only one of the combinations was used after the experimental conditions were established). The samples were put on ice immediately after the incubation. PhosSTOP and Complete Protease Inhibitor (Sigma-Aldrich PS/PI 4906837001/11697498001) solution was then added to stop digestion. The digestion solution was filtered through a $70 \mu \mathrm{m}$ cell strainer (Biosharp, BS-70-XBS) to remove larger tissue debris. The filtrate was processed by differential centrifugation ( $500 \times \mathrm{g}, 10 \mathrm{~min} ; 3,000 \times \mathrm{g}, 20 \mathrm{~min} ; 12,000 \times \mathrm{g}, 20 \mathrm{~min}$ ) to remove cellular debris, large vesicles, and some impurity particles. The obtained supernatant was centrifuged twice at $100,000 \times \mathrm{g}$ for $70 \mathrm{~min}$, and the precipitation was resuspended with PBS.

Temperature for all centrifugations was $4^{\circ} \mathrm{C}$. The above centrifugation procedure was referred as method 1. For the second method (Method 2), the samples were filtered with $0.22-\mu \mathrm{m}$ filters (Millipore, SLGPR33RB) to remove impurity particles and insoluble matter (Fig. 1c). Besides the 0.22- $\mu \mathrm{m}$ filtration process, the third method (method 3 ) also added a series of additional centrifugation steps to further remove some contaminations (Fig. 1d). During this process, a portion of the supernatant after completion of centrifugation $(3,000 \times \mathrm{g}, 12,000 \mathrm{xg})$ was taken and centrifuged again at the same centrifugal force for $3 \mathrm{~min}$. When aspirating the liquid, as little of the upper white layer as possible was recovered so as not to affect sEV purity. The precipitates produced by the differential ultracentrifugation steps of method 1 (i) and method 3 (ii) are shown in Fig. 1e. The whole separation scheme for tdsEVs and cedsEVs (Fig. 1b) was modified from the protocol established by Vella et al. (10) and Hoshino et al. (11).

\section{NanoFCM analysis}

NanoFCM with high throughput, high sensitivity and high resolution was used for the analysis of EVs (12; 13). The number of each $s E V$ sample was determined by calibrating the sample flow rate using a green fluorescent sphere with a known particle concentration $\left(1.9 \times 10^{10}\right.$ particles $\left./ \mathrm{mL}\right)$. Size distribution was calculated by standard curves generated using several nanomicrospheres of different diameters (68-155 $\mathrm{nm}$ ) under the same detection condition. Distribution histograms or dot-plots were derived from data collected at $1 \mathrm{~min}$ for all samples. For immunofluorescent staining, antibodies were purchased from BD 
Pharmingen $^{\text {TM }}$ : FITC Mouse Anti-Human CD9 (Clone M-L13), FITC Mouse Anti-Human CD63 (Clone H5C6). Add $20 \mu \mathrm{L}$ of FITC-labeled anti-CD9 and CD63 antibodies to $5 \mu \mathrm{L}$ of different sEV samples with particle concentration of approximately $1 \times 10^{10}$ particles $/ \mathrm{mL}$. These mixtures were incubated at $37^{\circ} \mathrm{C}$ for $30 \mathrm{~min}$. Care was taken to avoid light throughout. Then they were washed twice with PBS at 100,000 $\times \mathrm{g}$ for 30 min at $4^{\circ} \mathrm{C}$ (Beckman Coulter MAX-TL centrifuge, TLA-110 rotor). The pellet was resuspended in $100 \mu \mathrm{L}$ PBS for NanoFCM (N30E, NanoFCM Inc., China) analysis. 1\% Triton X-100 has been previously used for the assessment of EVs purity (12; 14). Triton X-100, as a nonionic surfactant, disrupts the phospholipid membrane. To calculate sEVs purity $(P)$, particle number before $(C 1)$ and after $(C 2)$ the treatment were enumerating, $\mathrm{P}=(\mathrm{C} 1-\mathrm{C} 2) / \mathrm{C} 1$. In detail, $5 \mu \mathrm{L}$ of $10 \%$ Triton $\mathrm{X}-100$ was added to $45 \mu \mathrm{L}$ of diluted sEVs, while a mixture of $5 \mu \mathrm{L}$ of PBS and $45 \mu \mathrm{L}$ of the sample was incubated on ice for $1 \mathrm{~h}$ as a control. The treated sEV samples were diluted 100-fold and used for NanoFCM assay.

\section{Transmission electron microscopy}

$10 \mu \mathrm{L}$ of sEVs sample was placed on formvar-/carbon-coated grid, which was cleaned in advance with a plasma surface treatment instrument (PDC-32G-2, Harrick Plasma, USA). The sample was allowed to settle for 10 min before stained with $2 \%$ phosphotungstic acid for $1 \mathrm{~min}$. Grids were imaged with a JEM 1200EX (Jeol Ltd, Japanese) transmission electron microscope operating at $120 \mathrm{kV}$.

\section{Western blot analysis}

The protein concentrations of sEV samples and cells were measured with Pierce ${ }^{\mathrm{TM}}$ BCA Protein Assay Kit (Product No. 23,227, Thermo Scientific, USA). Protein standard samples ranging from $0.0625 \mathrm{mg} / \mathrm{mL}$ to 1 $\mathrm{mg} / \mathrm{mL}$ were used. The protein content of each sample adjusted to $5 \mu \mathrm{g}$ was loaded onto a $12 \%$ polyacrylamide gel for electrophoresis. Proteins were then transferred from the gel to a PVDF membrane (MILLIPORE) using a constant current of $200 \mathrm{~mA}$ under low temperature. The membrane was blocked with $5 \%$ non-fat dry milk in TBST for $1 \mathrm{~h}$ at room temperature and incubated with primary antibody overnight at $4^{\circ} \mathrm{C}$. They were washed with $1 \times$ TBST, and incubated with horseradish peroxidase (HRP)conjugated secondary antibody at room temperature for $1 \mathrm{~h}$. The HRP-linked antibody was detected by incubation with New Cell \& Molecular Biotech's chemiluminescent substrate and the images were taken with JP-K300 (Shanghai JiaPeng Science technogy). The following antibodies used for immunoblotting were purchased from Abcam. Rabbit monoclonal anti-human CD9 antibody (Clone EPR2949, dilution 1:1000), rabbit monoclonal anti-human CD63 antibody (Clone EPR5702, dilution 1:1000), rabbit monoclonal anti-human TSG101 antibody (Clone EPR7130(B), dilution 1:1000), rabbit monoclonal antihuman HSP70 antibody (Clone EPR2914, dilution 1:1000). The following antibodies were purchased from Proteintech. Mouse monoclonal anti-human GM130 antibody (Clone 2A4F11, dilution 1:1000) and the secondary antibodies: horseradish peroxidase-labelled goat anti-rabbit and goat anti-mouse.

\section{Statistical analysis}

Data were analyzed with GraphPad Prism version 8.0 (GraphPad Software). ANOVA and paired two-tailed Student's t test were applied to test differences in sEV samples. Differences with $P<0.05$ were considered 
statistically significant. GraphPad Software was used for calculation of the mean and the standard error of mean (SEM). Figures were prepared using GraphPad Software, Photoshop and FlowJo.

\section{Results}

\section{Establishment of isolation and purification methods}

Figure $2 \mathrm{a}$ shows that the particle concentrations obtained by method 1 were significantly higher for the 20(1) and 30(1) groups compared to the other two methods, and the protein content increased significantly except for the 30(1) group (Fig. 2b). However, the data reproducibility was poor, which suggests that the sEV samples obtained by method 1 may contain more impurity particles and heteroproteins. The particle number and protein content of $s E V s$ obtained from method 2 were not significantly different from those of method 3 but were lower than those of method 1 (Fig. 2a,b). TdsEVs and cedsEVs isolated by ultracentrifugation were susceptible to contamination with non-vesicular matter such as lipoproteins. $1 \%$ of Triton X-100 was used to evaluate the purity of sEV. Treatment of samples with the detergent resulted in rupture of the $\mathrm{SEV}$ s, while non-membranous contaminants remained intact (15). Typical SSC burst traces (c) and SSC burst area distribution histograms ( $d$ and e) are presented in Fig. 2, which exhibit the graphical changes before and after treatment with $1 \%$ Triton X-100 for tdsEVs with high purity (89.53\%). A significant decrease in the event rate and the number of particles detected per minute can be observed after the treatment. Using this approach, we examined the purity of sEV derived from three methods (Figure S1) and found that the values of method 1 and method 2 were essentially negative (Fig. 2f), which indicates that the number of particles after the treatment exceeds the number before the treatment. Tian et al. tested very low density lipoprotein (VLDL) by NanoFCM after treating it with Triton X-100(14). Particle number after treatment was more than twice as large as that before treatment. Therefore, the sEVs derived from the first two methods may be contaminated with lipoproteins such as VLDL. The purity of sEVs obtained by method 3 was much higher than those of method 1 and method 2 (Fig. 2f). Among them, the mean values of sEV purity for each group were $86.89 \%, 83.3 \%, 73.46 \%, 11.48 \%, 28.9 \%$, and $60.71 \%$, respectively. Also, it is noteworthy that the sEVs obtained by ultracentrifugation are prone to form aggregation (16). The aggregates may be dispersed after Triton X-100 treatment, changing from clusters of vesicles with larger particle size to multiple small vesicles. Hence, the actual purity of $\mathrm{SEV}$ was expected to be higher than the measured results.

\section{Determination of incubation concentrations and times for collagenase D and DNase I}

NanoFCM was able to analyze the surface proteins of EVs by immunofluorescent labeling (14). Using a NanoFCM-based approach, two markers of EVs, CD9 and CD63 (17), were analyzed to determine the optimal combination of enzyme concentration and incubation time. CD81 was not used as a reference because of the interference of digestive enzymes (9). Figure 3a depicts typical burst traces of tdsEVs and PBS. The blue rectangular boxes of the SSC histograms mark some particles of larger size present in the PBS or samples (Fig. 3b). The black dots in the bivariate dot-plots represent particles without 
fluorescence or impurity particles. The mean values of proportion of CD9 in six groups were $15.7 \%, 9.9 \%$, $9.43 \%, 4.16 \%, 5.38 \%$, and $5.01 \%$, respectively. The $20(1)$ group was significantly higher than the other groups (Fig. 3c,e). In contrast, no similar phenomenon was observed in CD63 with mean values of 16.8\%, $15.63 \%, 12.57 \%, 8.87 \%, 9.97 \%, 7.55 \%$, respectively (Fig. 3d,e). Western blot showed a similar trend on CD9 with NonoFCM analysis and GM130 was negatively expressed on tdsEV (Fig. 3f). Therefore, based on the protein content of CD9, we chose 20(1) as the condition for subsequent experiments. That is, the enzyme concentrations of collagenase $D$ and DNase I were $4 \mathrm{mg} / \mathrm{mL}$ and $80 \mathrm{U} / \mathrm{mL}$, and the incubation time was $20 \mathrm{~min}$.

\section{Particle concentration, size, protein content and purity analysis of sEVs prepared from the three different methods}

Based on the above findings, we compared the differences among cedsEVs, fresh and frozen tdsEVs. In these experiments, NanoFCM analysis showed that the particle concentration of cedsEVs was significantly lower than fresh $(p=0.0037)$ and frozen tdsEVs $(p=0.0022)$, while there was no significant difference between fresh and frozen tdsEVs (Fig. 4a). The BCA results showed that protein content of cedsEVs was significantly lower than that of fresh tdsEVs $(p=0.0041)$, while no significant difference was observed between fresh and frozen tdsEVs (Fig. 4b). When the sizes of these sEVs were compared, it was found that cedsEVs were significantly smaller than fresh $(p=0.0011$ and 0.0032$)$ and frozen tdsEVs $(p=0.0053$ and 0.0084 ), while there was no significant difference between fresh and frozen tdsEVs (Fig. 4c). The particle: protein ratio is commonly used as a surrogate marker for preparation purity of EVs (18; 19). Figure $4 d$ shows that the ratio of cedsEVs was significantly lower than those of fresh $(p=0.013)$ and frozen tdsEVs $(p=0.0017)$, while those of fresh and frozen tdsEVs were not significantly different. This is in general agreement with the results of sEV purity assessment by $1 \%$ Triton X-100 (Fig. 4e). The typical vesicle morphology is shown in Fig. 4f, which suggests that cedsEVs had fewer vesicles and smaller particle size than tdsEVs. These results are consistent with the NonoFCM analysis. In summary, fresh tdsEVs were significantly higher than cedsEVs in terms of particle number, protein content. They also had a better size distribution and purity. On the other hand, fresh and frozen tdsEVs were not significantly different from each other.

\section{Comparison of protein markers for three types of sEV}

Figure 5 demonstrates FITC fluorescence versus SSC for cedsEVs, fresh and frozen tdsEVs were labeled with FITC-conjugated mAbs specific to CD9 (a) and CD63 (b), while PBS as a blank control is not deducted. The mean values for the CD63 of three groups were $13 \%, 8.32 \%$, and $6.84 \%$, respectively. CedsEVs were significantly higher than fresh $(p=0.046)$ and frozen tdsEVs in the fluorescent intensity ( $p$ $=0.0145$ ), but there was no significant between fresh and frozen tdsEVs (Fig. $5 \mathrm{c}$ ). The results were consistent with western blot (Fig. 5e). For CD9, the mean values were $4.09 \%, 3.9 \%$ and $4.31 \%$. No significant difference among them was found (Fig. 5d). However, western blot showed higher protein content of CD9 in fresh and frozen tdsEVs than in cedsEVs (Fig. 5e). Similarly, two EVs markers of 
cytoplasmic protein, TSG101 and HSP70, exhibited the same trend as that of CD9. GM130 was utilized as a negative control marker for SEVs (17), which was present in the cell extracts but absent in cedsEVs, fresh and frozen tdsEVs. Coomassie blue staining (Fig. 5f) indicated that cedsEVs contained fewer protein species than fresh and frozen tdsEVs which had similar protein stripes. Meanwhile, fresh tdsEVs appeared to contain more proteins than frozen tdsEVs.

\section{Discussion}

Currently, there is a tremendous interest in developing biomarkers from tdsEVs and cedsEVs for diagnosis of cancers. Both sEVs obtained from the interstitial space of tissues (20) and cultivation of tissue explants (11) have promising potential to be developed as biomarkers. Commonly, the methods for enriching sEVs from tissues are sucrose density gradient ultracentrifugation (SDGU) (21) and iodixanol density gradient ultracentrifugation (8). While the purity of EVs obtained with these centrifugation methods is high, the purification process is time-consuming and requires complex experimental procedures and techniques, which makes the methods impractical for clinical diagnosis. In the present study, we examined and compared sEVs obtained by different isolation methods in terms of the particle concentration, protein content, size distribution, purity, and conventional protein markers. Based on these analyses, we developed a simple method (method 3 ) for isolation of sEVs with high purity using human liver cancer tissues.

Notably, this method has many advantages including low cost, short time and low operating requirements for researchers. Meanwhile, the mean purity (86.89\%) of sEVs derived from the optimal combination of incubation time and enzyme concentration was higher than that of EVs extracted by ultracentrifugation method in platelet-free plasma (77.8\%) (14) and even comparable to the purity (85\%-90\%) of EVs derived from cell lines of more homogeneous origin (12). Furthermore, in comparison with SDGU, the conventional isolation and purification method (20), this newly developed method exhibited better EVs enrichment effects. In terms of particle concentration and protein content, the method (approximately $1 \times 10^{10}$ particles/100 mg tissue; $10 \mu$ g protein/100 mg tissue) was higher than SDGU (approximately $1 \times 10^{9}$ particles/100 mg tissue; $6 \mu$ protein/100 $\mathrm{mg}$ tissue). In the particle: protein ratio, our method (approximately $8 \times 10^{8}$ particles/ $\mu$ g protein) was greater than SDGU (approximately $1 \times$ $10^{8}$ particles $/ \mu$ g protein). Although inconsistency of the subjects may contribute to the bias of experimental results. Nevertheless, we believe that this is not a major factor, and more experiments need to be conducted to validate the conclusion. It is worth noting that cedsEVs have lower purity and smaller in particle diameter compared to tdsEVs. We considered that cedsEVs may contain some small impurities which could not be removed through $0.22-\mu \mathrm{m}$ filters.

Some markers of EVs were used for comparison among cedsEVs, fresh and frozen tdsEVs. NanoFCM assay showed no significant difference for the expression of sEV surface protein CD9, which is not consistent with western blot. There are two potential reasons: (1) CD9 protein presents in non-EV materials; (2) TdsEVs and cedsEVs are acquired in different ways, and their sorting mechanisms are presumably different. Therefore, some differences may be existing between membrane and intracellular 
of sEVs. Coomassie blue staining reveals that cedsEVs have fewer protein species than other groups, but contain some unknown proteins of small molecular weights. Additional analyses, such as proteomics, are needed to identify the different protein species between cedsEVs and tdsEVs.

In conclusion, we developed a new and convenient method for the isolation and purification of sEVs and conducted experiments with liver cancer tissues to determine the appropriate enzyme concentration (collagenase D: $4 \mathrm{mg} / \mathrm{mL}$, DNase I: $80 \mathrm{U} / \mathrm{mL}$ ) and incubation time (20 min). This method established with liver cancer tissues may be applicable to other tissues, but it still needs to be explored. Furthermore, tdsEVs had larger particle size, higher yield and purity than cedsEVs. If researchers are interested in CD63, the choice of cedsEVs could be more appropriate. If one wants to explore the abundant protein species, tdsEVs may be a great choice.

\section{Declarations}

\section{Data Availability}

All data generated or analyzed during this study are included in the article.

\section{Acknowledgements}

The authors would like to thank the First Affiliated Hospital of Gannan Medical University for providing clinical samples.

\section{Funding}

This work was supported by the National Natural Science Foundation of China [grant numbers 81702580], Key R\&D Planning Project of Jiangxi Science and Technology Commission, China [No. 20203BBGL73126].

\section{Author information}

Jie Chen, Zhigang Jiao and Jianwen Mo contributed equally to this work.

\section{Affiliations}

\section{The First School of Clinical Medicine, Gannan Medical University, Ganzhou, China}

Jie Chen, Defa Huang, Zhengzhe Li, Tong Yang, Minghong Zhao, Fangfang Xie, Die Hu, Xiaoxing Wang, Xiaomei Yi \& Tianyu Zhong

\section{Laboratory Medicine, First Affiliated Hospital of Gannan Medical University, Ganzhou, China}

Jie Chen, Zhigang Jiao, Defa Huang, Zhengzhe Li, Wenjuan Zhang, Tong Yang, Minghong Zhao, Fangfang Xie, Die Hu, Xiaoxing Wang, Xiaomei Yi \& Tianyu Zhong 
Department of Orthopedic Surgery, First Affiliated Hospital of Gannan Medical University, Ganzhou, China

Jianwen Mo

Precision Medicine Center, First Affiliated Hospital of Gannan Medical University, Ganzhou, China

Zhigang Jiao, Wenjuan Zhang \& Tianyu Zhong

Department of Pharmacology and Chemical Biology, University of Pittsburgh School of Medicine, Pittsburgh, United States

Yu Jiang

\section{Contributions}

Conceived and Designed Experiments: JC, ZgJ, JwM, TyZ. Conducted experiments, analyzed and interpreted data: JC, JwM, DfH, ZgJ, ZzL, WjZ, TY, MhZ, FfX, DH, XxW, XmY, TyZ; Writing - draft preparation, review and editing: YJ, TyZ; Supervision: TyZ; Funding acquisition: TyZ.

\section{Corresponding author}

Tianyu Zhong, Laboratory Medicine, First Affiliated Hospital of Gannan Medical University, Ganzhou, China; Email: zhongtianyu@gmail.com

Ethics declarations

Conflict of Interest

The authors declare that they have no conflict of interest.

\section{Ethics Approval and Consent to Participate}

Informed written consents were obtained from the patients and the collection of human tissue samples was approved by the Ethics Committee of the First Affiliated Hospital of Gannan Medical University.

\section{Consent to Publish}

Not applicable

\section{References}

1. Zebrowska, A., Widlak, P., Whiteside, T., \& Pietrowska, M. (2020). Signaling of Tumor-Derived sEV Impacts Melanoma Progression. Int J Mol Sci, 21(14).

2. Takov, K., Yellon, D. M., \& Davidson, S. M. (2019). Comparison of small extracellular vesicles isolated from plasma by ultracentrifugation or size-exclusion chromatography: yield, purity and functional 
potential. J Extracell Vesicles, 8(1), 1560809

3. Erdbrugger, U., Blijdorp, C. J., Bijnsdorp, I. V., Borras, F. E., Burger, D., Bussolati, B., \& Martens-Uzunova, E. S. (2021). Urinary extracellular vesicles: A position paper by the Urine Task Force of the International Society for Extracellular Vesicles. J Extracell Vesicles, 10(7), e12093

4. Dixon, C. L., Sheller-Miller, S., Saade, G. R., Fortunato, S. J., Lai, A., Palma, C., \& Menon, R. (2018). Amniotic Fluid Exosome Proteomic Profile Exhibits Unique Pathways of Term and Preterm Labor. Endocrinology, 159(5), 2229-2240

5. Moller, A., \& Lobb, R. J. (2020). The evolving translational potential of small extracellular vesicles in cancer. Nat Rev Cancer, 20(12), 697-709

6. Yang, W. S., Zeng, X. F., Liu, Z. N., Zhao, Q. H., Tan, Y. T., Gao, J., \& Xiang, Y. B. (2020). Diet and liver cancer risk: a narrative review of epidemiological evidence. Br J Nutr, 124(3), 330-340

7. Crescitelli, R., Lasser, C., Jang, S. C., Cvjetkovic, A., Malmhall, C., Karimi, N., \& Lotvall, J. (2020). Subpopulations of extracellular vesicles from human metastatic melanoma tissue identified by quantitative proteomics after optimized isolation. J Extracell Vesicles, 9(1), 1722433

8. Jang, S. C., Crescitelli, R., Cvjetkovic, A., Belgrano, V., Bagge, O., Sundfeldt, R., \& Lotvall, K., J. (2019). Mitochondrial protein enriched extracellular vesicles discovered in human melanoma tissues can be detected in patient plasma. J Extracell Vesicles, 8(1), 1635420

9. Crescitelli, R., Lasser, C., \& Lotvall, J. (2021). Isolation and characterization of extracellular vesicle subpopulations from tissues. Nat Protoc, 16(3), 1548-1580

10. Vella, L. J., Scicluna, B. J., Cheng, L., Bawden, E. G., Masters, C. L., Ang, C. S., \& Hill, A. F. (2017). A rigorous method to enrich for exosomes from brain tissue. J Extracell Vesicles, 6(1), 1348885

11. Hoshino, A., Kim, H. S., Bojmar, L., Gyan, K. E., Cioffi, M., Hernandez, J., \& Lyden, D. (2020). Extracellular Vesicle and Particle Biomarkers Define Multiple Human Cancers. Cell, 182(4), 104410611018

12. Tian, Y., Ma, L., Gong, M., Su, G., Zhu, S., Zhang, W., \& Yan, X. (2018). Protein Profiling and Sizing of Extracellular Vesicles from Colorectal Cancer Patients via Flow Cytometry. ACS Nano, 12(1), 671680

13. Dooley, K., McConnell, R. E., Xu, K., Lewis, N. D., Haupt, S., Youniss, M. R., \& Williams, D. E. (2021). A versatile platform for generating engineered extracellular vesicles with defined therapeutic properties. Mol Ther, 29(5), 1729-1743

14. Tian, Y., Gong, M., Hu, Y., Liu, H., Zhang, W., Zhang, M., \& Yan, X. (2020). Quality and efficiency assessment of six extracellular vesicle isolation methods by nano-flow cytometry. $J$ Extracell Vesicles, 9(1), 1697028

15. Osteikoetxea, X., Sodar, B., Nemeth, A., Szabo-Taylor, K., Paloczi, K., Vukman, K. V., \& Buzas, E. I. (2015). Differential detergent sensitivity of extracellular vesicle subpopulations. Org Biomol Chem, 13(38), 9775-9782

16. Dash, M., Palaniyandi, K., Ramalingam, S., Sahabudeen, S., \& Raja, N. S. (2021). Exosomes isolated from two different cell lines using three different isolation techniques show variation in physical and 
molecular characteristics. Biochim Biophys Acta Biomembr, 1863(2), 183490

17. Théry, C., Witwer, K. W., Aikawa, Elena, Alcaraz, M., Jose, Anderson, J. D., \& Andriantsitohaina, Ramaroson,... Zuba-Surma, E. K. (2018). Minimal information for studies of extracellular vesicles 2018 (MISEV2018): a position statement of the International Society for Extracellular Vesicles and update of the MISEV2014 guidelines. Journal of Extracellular Vesicles, 7(1).

18. Woo, H. K., Sunkara, V., Park, J., Kim, T. H., Han, J. R., Kim, C. J., \& Cho, Y. K. (2017). Exodisc for Rapid, Size-Selective, and Efficient Isolation and Analysis of Nanoscale Extracellular Vesicles from Biological Samples. ACS Nano, 11(2), 1360-1370

19. Webber, J., \& Clayton, A. (2013). How pure are your vesicles? J Extracell Vesicles, 2.

20. Huang, Y., Cheng, L., Turchinovich, A., Mahairaki, V., Troncoso, J. C., Pletnikova, O., \& Witwer, K. W. (2020). Influence of species and processing parameters on recovery and content of brain tissuederived extracellular vesicles. J Extracell Vesicles, 9(1), 1785746

21. Muraoka, S., DeLeo, A. M., Sethi, M. K., Yukawa-Takamatsu, K., Yang, Z., Ko, J., \& Ikezu, T. (2020). Proteomic and biological profiling of extracellular vesicles from Alzheimer's disease human brain tissues. Alzheimers Dement, 16(6), 896-907

\section{Figures}
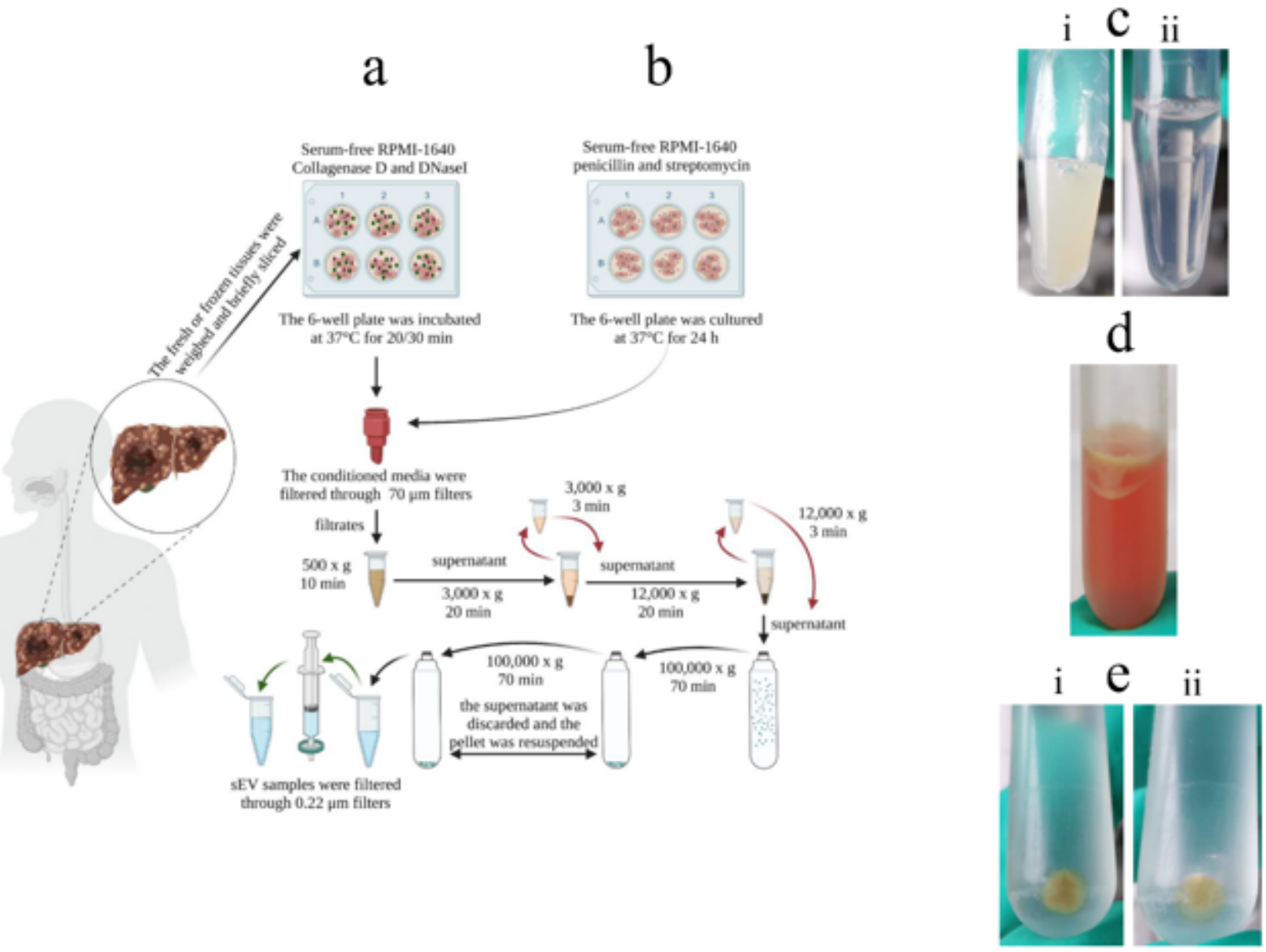
Figure 1

Schematic overview of enrichment methods for tdsEVs and cedsEVs. (a) Three different methods for the isolation and purification of tdsEVs. The black arrows are the processes they share, the green arrows are the additional steps common to method 2 and method 3 , and the red arrows represent the added steps of method 3. (b) Method used for preparation of cedsEVs. The different color arrows are as in (a). (c) Comparison of before (i) and after (ii) filtration of tdsEVs through $0.22-\mu \mathrm{m}$ filters. (d) White contaminants in the supernatant. (e) The pellets produced by differential ultracentrifugation steps of method 1 (i) and method 3 (ii).
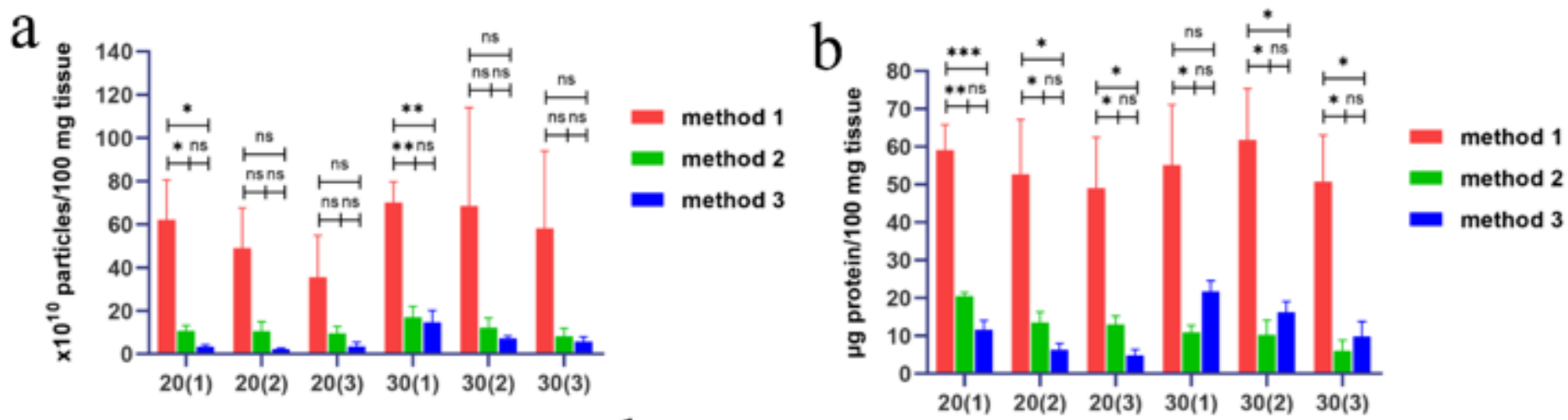

c
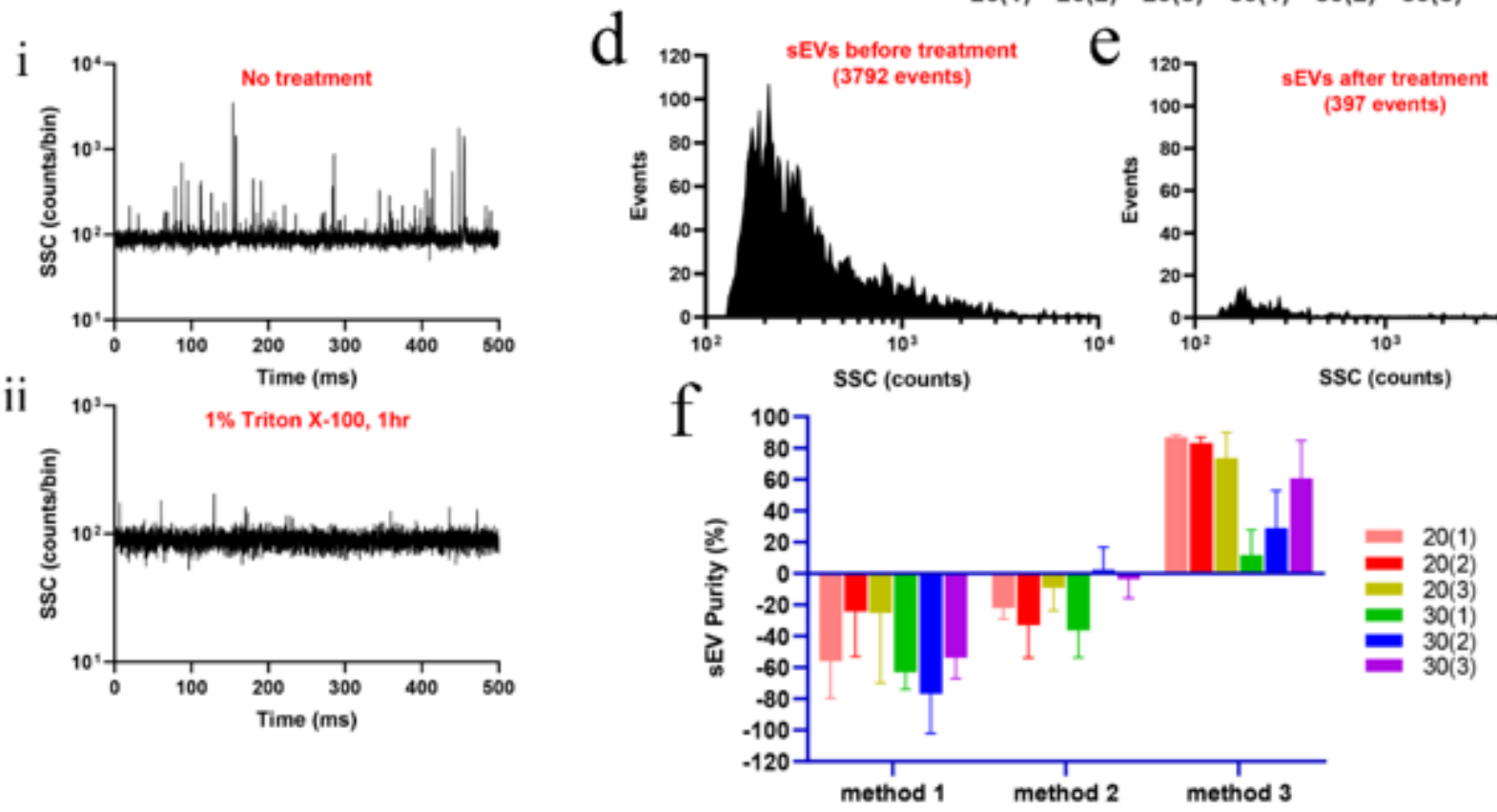

\section{Figure 2}

Particle concentration, protein content and purity analysis of the three different methods for separation of tdsEVs. (a) Particle concentrations of tdsEVs isolated by three methods at different combinations of enzyme concentrations and incubation times were measured by NanoFCM. The results for each group were normalized by tissue weight (per $100 \mathrm{mg}$ ). (1), (2) and (3) represent the concentration combinations of collagenase $D(4 \mathrm{mg} / \mathrm{mL}, 2 \mathrm{mg} / \mathrm{mL}$ and $1 \mathrm{mg} / \mathrm{mL})$ and DNase I $(80 \mathrm{U} / \mathrm{mL}, 40 \mathrm{U} / \mathrm{mL}$ and $20 \mathrm{U} / \mathrm{mL})$. $20 / 30$ is the incubation time of $20 / 30 \mathrm{~min}$. For instance, $20(1)$ is collagenase $D(4 \mathrm{mg} / \mathrm{mL}$ ), DNase I ( 80 
$\mathrm{U} / \mathrm{mL}$ ) and incubation time (20 $\mathrm{min}$ ). (b) Protein concentrations of tdsEVs separated by different methods in the different combinations, which were determined by the BCA protein assay (per $100 \mathrm{mg}$ tissue). (a-b): data are shown as mean \pm SEM. ${ }^{\star} p<0.05,{ }^{\star *} p<0.01,{ }^{* \star *} p<0.001$ by paired two-tailed Student's $t$ test. (c) Representative SSC burst traces of tdsEVs preparation by method 3 before (i) and after (ii) $1 \%$ Triton X-100 treatment for $1 \mathrm{~h}$ on ice; (d and e) SSC distribution histograms of tdsEVs preparation by method 3 before (d) and after (e) Triton X-100 treatment. (f) Purity measurement for tdsEVs in the different combinations $(n=3$, mean \pm SEM).
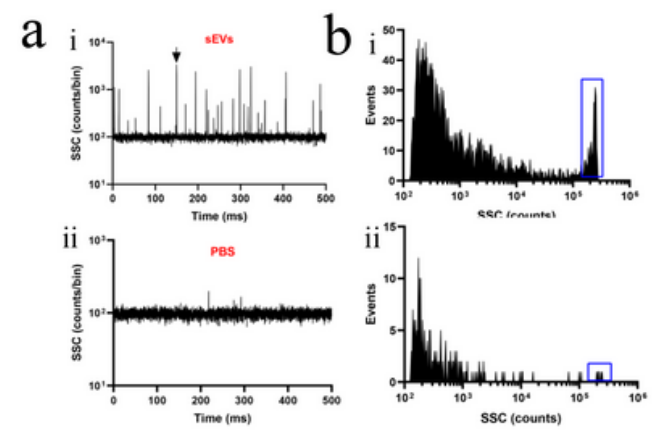

$\mathrm{e}$

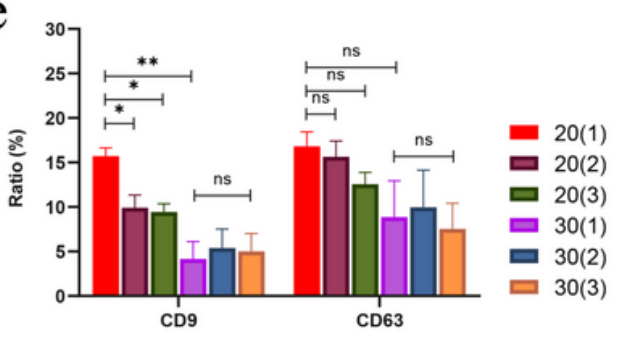

f

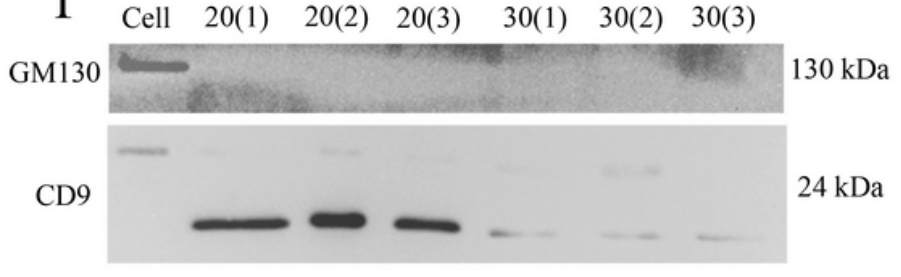

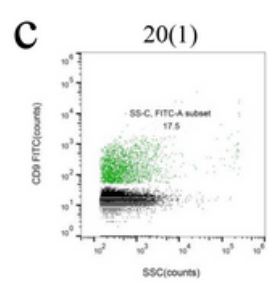

30(1)
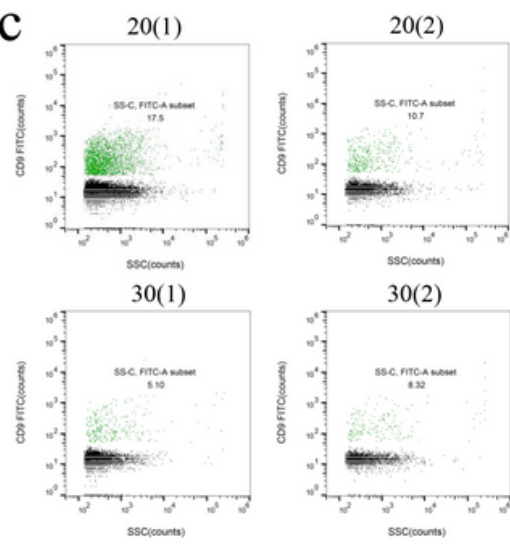

30(2)
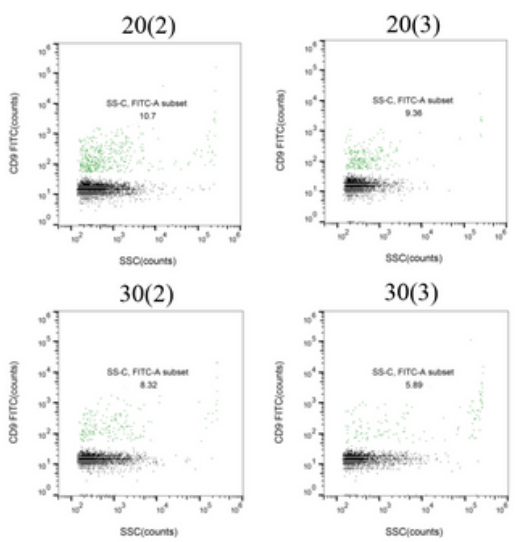

30(3)
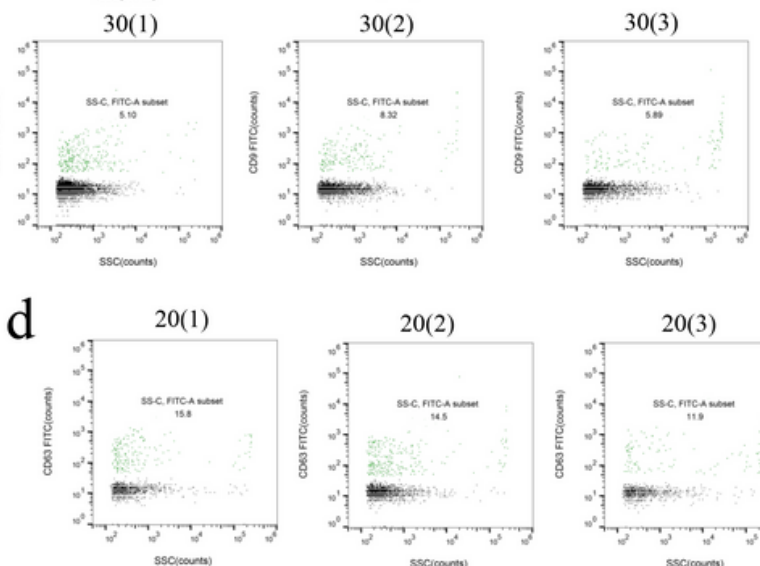

30(1)

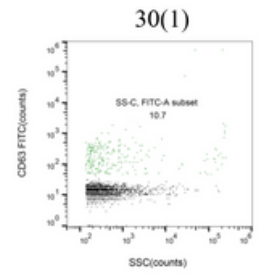

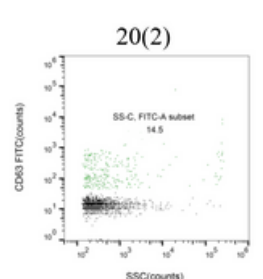

$30(2)$

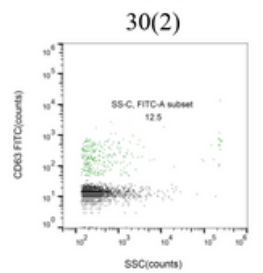

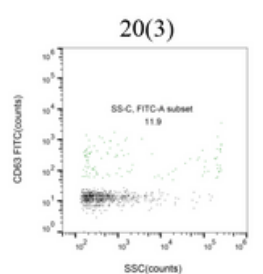

30(3)

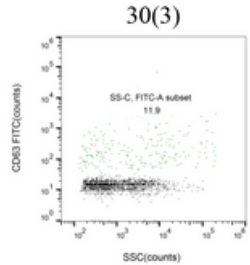

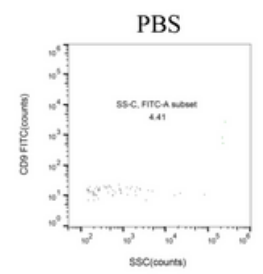

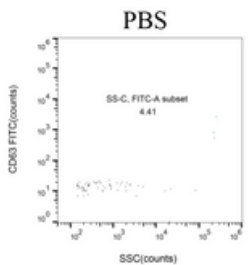

Figure 3

Investigation of conditions for separation of tdsEVs by NanoFCM. (a) Representative SSC burst traces of tdsEVs preparation by method 3 (i) and PBS (ii); (b) SSC distribution histograms of the SEVs (i) and PBS (ii) derived from data collected over 1 min each. (c and d) Bivariate dot-plots of FITC fluorescence versus SSC for tdsEVs preparation by method 3 and PBS. The sEVs and PBS were labeled with FITC-conjugated mAbs specific to CD9 (i) and CD63 (ii). The percentages of phenotype-positive sEVs are provided in each plot. (e) Measured percentages of a specific phenotype-positive sEVs $(n=3)$ for CD9 and CD 63 of the different combinations were analyzed by paired two-tailed Student's $t$ test and expressed as mean \pm SEM. ${ }^{*} p<0.05,{ }^{*} p<0.01$ and ns: no significant difference. (f) Western blot analysis of CD 9 and GM130 associated with cell and $s E V$ from the different combinations $(n=3,5 \mu$ g protein was loaded per lane). 


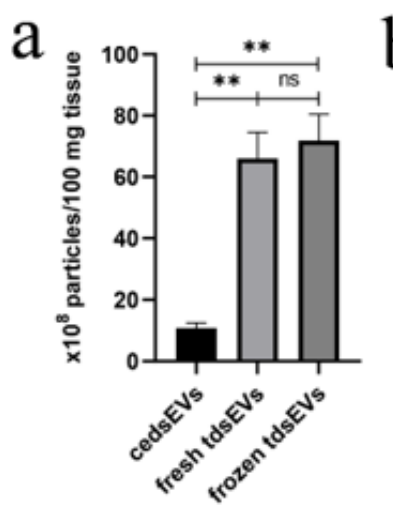

b
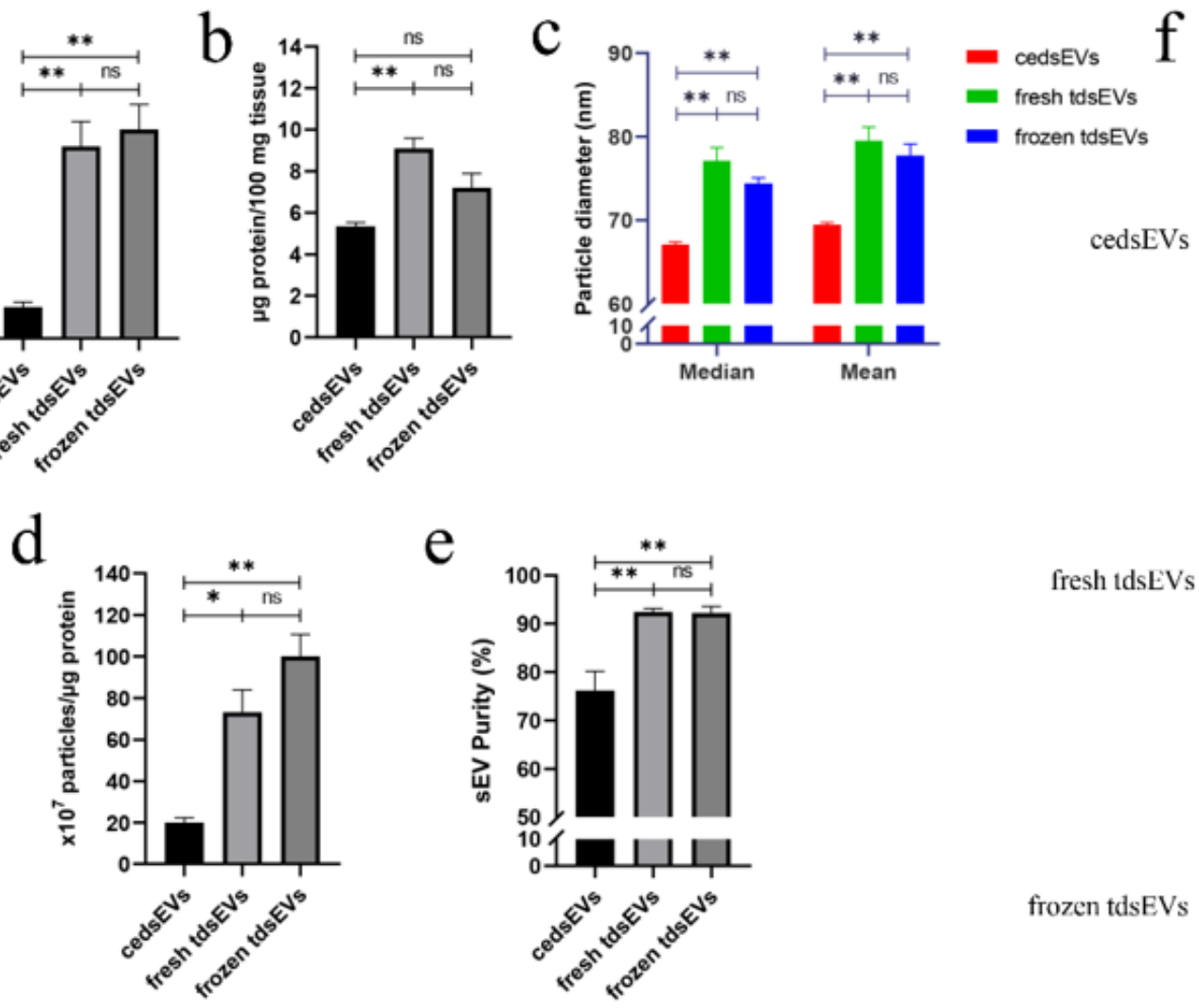

fresh tdsEVs
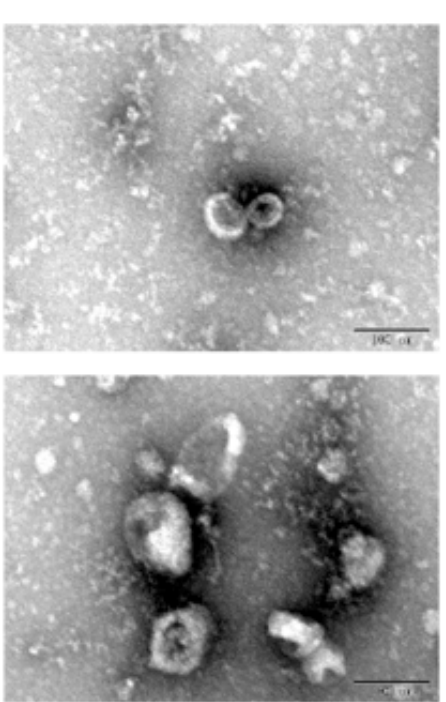

frozen tdsEVs

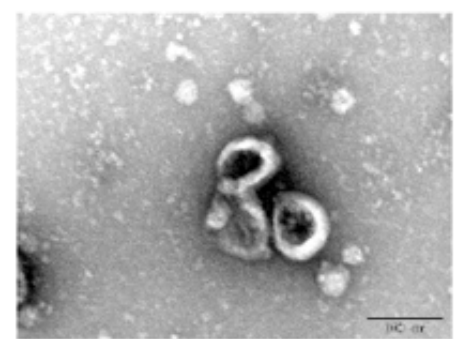

\section{Figure 4}

Particle number, protein content, diameter distribution and purity of cedsEVs, fresh and frozen tdsEVs. (a) The particle number of isolated fractions was measured by NanoFCM. Particle concentrations in each group were normalized by tissue weight (per $100 \mathrm{mg}$ ). (b) The total protein content of isolated particles in three groups was measured by BCA assay (per $100 \mathrm{mg}$ tissue). (c) Median and mean values of particle diameter distribution for three groups. (d) The particle number/ $\mu$ g protein ratio for three groups. (e) Purity measurement for three groups by $1 \%$ Triton X-100. (a-e) $n=3,{ }^{\star} p<0.05,{ }^{*} p<0.01$ by ANOVA test. (f) CedsEVs, fresh and frozen tdsEVs were visualized by negative staining transmission electron microscopy (scale bar $=100 \mathrm{~nm}$ ) which is representative of five images taken of each fraction. 

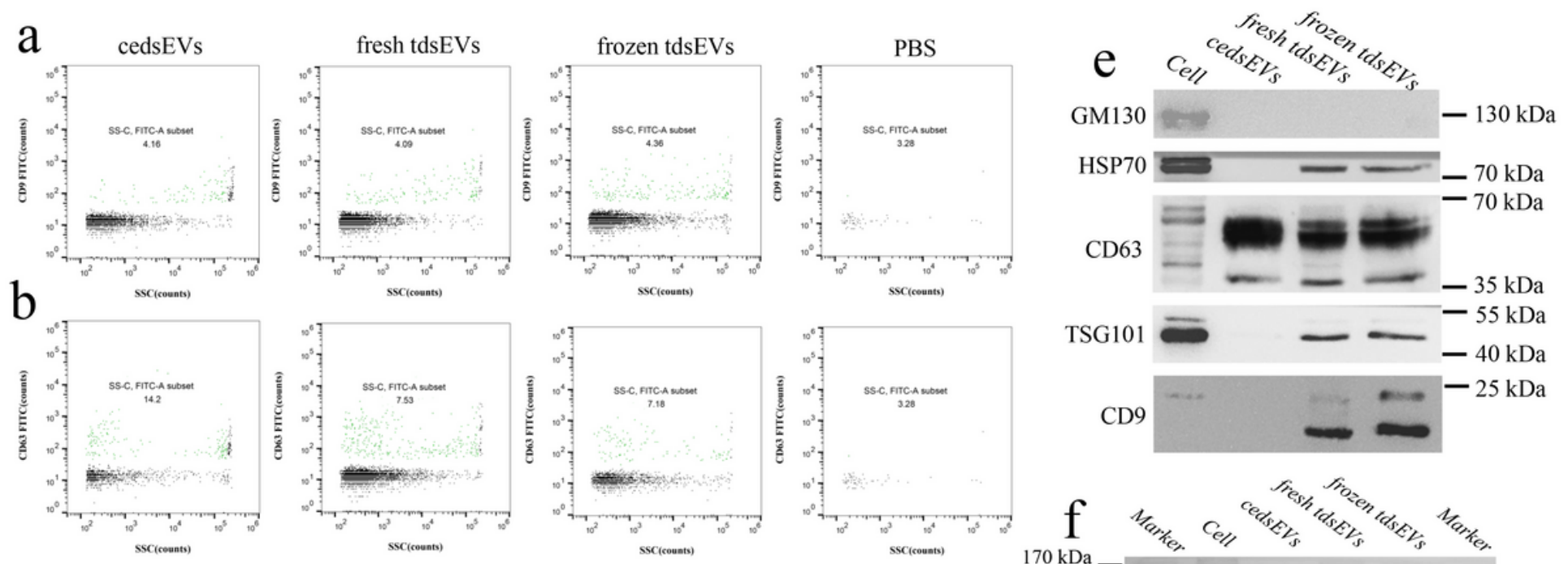

c
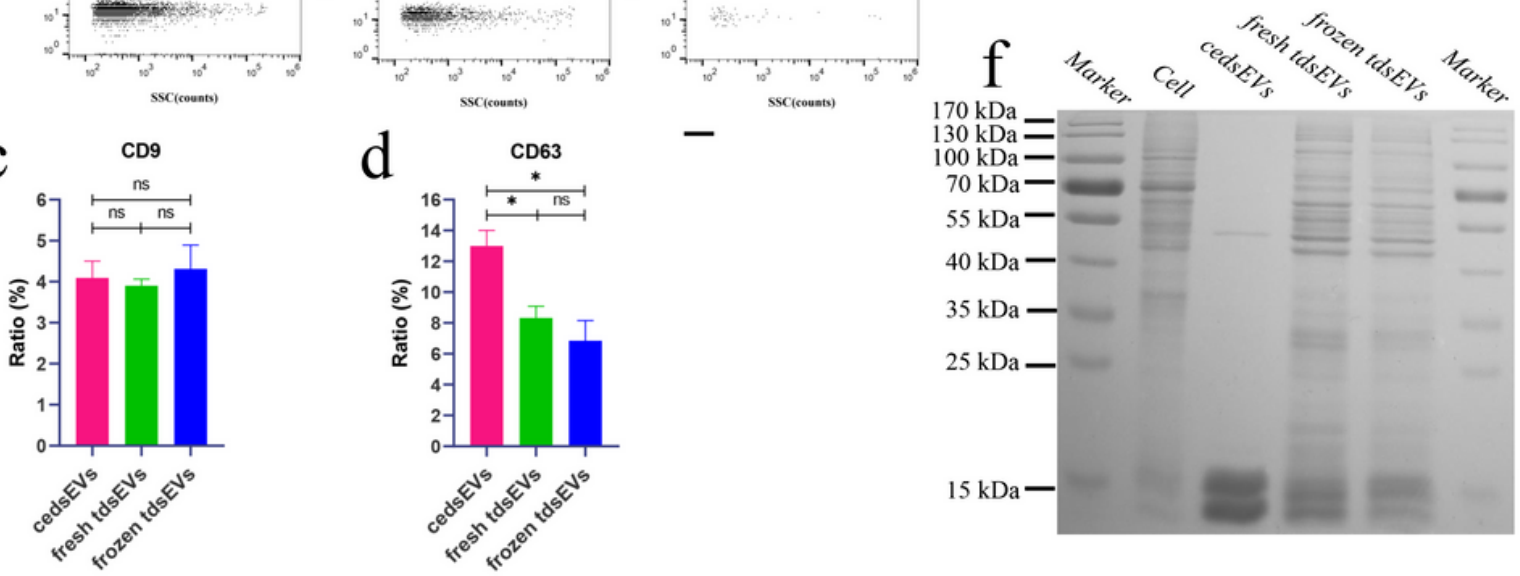

\section{Figure 5}

Various markers were used for the comparison of cedsEVs, fresh and frozen tdsEVs. ( $a$ and $b$ ) Bivariate dot-plots of FITC fluorescence versus SSC for three types of SEVs and PBS were labeled with FITCconjugated mAbs specific to CD9 (a) and CD63 (b). The percentages of phenotype-positive sEVs for CD9 and CD63 are shown in each plot. (c and d). Measured percentages of a specific phenotype-positive sEVs $(n=3)$ for CD9 and CD63 of three types of sEVs (mean \pm SEM). ${ }^{*} p<0.05$ by ANOVA test. (e) Western blot analysis of GM130, HSP70, CD63, TSG101 and CD9 in cell extracts and the three types of sEVs. (f) Coomassie blue staining of the total proteins from cell extracts and three types of sEVs. (e and f). $5 \mu \mathrm{g}$ protein was loaded per lane.

\section{Supplementary Files}

This is a list of supplementary files associated with this preprint. Click to download.

- GraphicalAbstract.png

- supplementarymaterial.rar 